\title{
Phase separation and exchange biasing in the ferromagnetic IV-VI semiconductor $\mathrm{Ge}_{1-x} \mathrm{Mn}_{x} \mathrm{Te}$
}

\author{
R. T. Lechner, ${ }^{1,2, a)}$ G. Springholz, ${ }^{1}$ M. Hassan, ${ }^{1}$ H. Groiss, ${ }^{1}$ R. Kirchschlager, ${ }^{1}$ J. Stangl, ${ }^{1}$ \\ N. Hrauda, ${ }^{1}$ and G. Bauer ${ }^{1}$ \\ ${ }^{1}$ Institut für Halbleiter- und Festkpörperphysik, Johannes Kepler Universität Linz, 4040 Linz, Austria \\ ${ }^{2}$ Institute of Physics, University of Leoben, 8700 Leoben, Austria
}

(Received 14 April 2010; accepted 10 June 2010; published online 12 July 2010)

\begin{abstract}
Ferromagnetic $\mathrm{Ge}_{1-x} \mathrm{Mn}_{x} \mathrm{Te}$ grown by molecular beam epitaxy with Mn content of $x_{\mathrm{Mn}} \approx 0.5$ is shown to exhibit a strong tendency for phase separation. At higher growth temperatures apart from the cubic $\mathrm{Ge}_{0.5} \mathrm{Mn}_{0.5} \mathrm{Te}$, a hexagonal MnTe and a rhombohedral distorted $\mathrm{Ge}_{0.83} \mathrm{Mn}_{0.17} \mathrm{Te}$ phase is formed. This coexistence of antiferromagnetic MnTe and ferromagnetic $\mathrm{Ge}_{0.5} \mathrm{Mn}_{0.5} \mathrm{Te}$ results in magnetic exchange-bias effects. (c) 2010 American Institute of Physics. [doi:10.1063/1.3459149]
\end{abstract}

Transition metal doped semiconductors, which combine ferromagnetic and semiconducting properties, have drawn tremendous interest in the past few years for possible future spintronic applications. ${ }^{1,2}$ Among the so far less explored materials are magnetic IV-VI compounds ${ }^{6}$ like $\mathrm{Ge}_{1-x} \mathrm{Mn}_{x} \mathrm{Te}$, for which ferromagnetic Curie temperatures $T_{\mathrm{C}}$ of $\approx 150 \mathrm{~K}$ have been reported for bulk material ${ }^{3}$ and up to $190 \mathrm{~K}$ for epitaxial layers grown by molecular beam epitaxy (MBE). ${ }^{4,5}$ In $\mathrm{Ge}_{1-x} \mathrm{Mn}_{x} \mathrm{Te}$ the incorporated $\mathrm{Mn}^{2+}$ is isoelectronic to $\mathrm{Ge}$ and therefore, the carrier concentration can be controlled independently of the Mn content. ${ }^{7}$ Moreover, the solubility limit of $\mathrm{Mn}$ in the GeTe host lattice is rather large and reaches up to about $60 \%$ in single-phase bulk material $\mathrm{Ge}_{1-x} \mathrm{Mn}_{x} \mathrm{Te}^{3,8}$ Between $x_{\mathrm{Mn}}=60$ to $90 \%, \mathrm{Ge}_{1-x} \mathrm{Mn}_{x} \mathrm{Te}$ is two-phase and exists in the cubic $\mathrm{NaCl}$ and hexagonal NiAs phase. ${ }^{3,8}$ This is due to the different crystal structures of the alloy constituents, where GeTe is ferroelectric and crystallizes in a rhombohedrally distorted rock salt lattice ${ }^{3}$ and bulk MnTe in the hexagonal NiAs structure ${ }^{9}$ but can be also stabilized in the cubic zinc blende structure in epitaxial layers. ${ }^{10}$ Both MnTe phases are antiferromagnetic (AFM) with Néel temperatures $T_{\mathrm{N}}=310 \mathrm{~K}$ and $65 \mathrm{~K}$, respectively. ${ }^{9,10}$

In this Letter, the structural and magnetic properties of $\mathrm{Ge}_{1-x} \mathrm{Mn}_{x} \mathrm{Te}$ epilayers with Mn content close to the solubility limit are investigated. The samples were grown by MBE onto $\mathrm{BaF}_{2}$ (111) substrates and the $\mathrm{Mn}$ content adjusted in the range of 45 to $55 \%$. It is shown that for these high $\mathrm{Mn}$ contents a pronounced tendency for phase separation exists. In particular, at higher growth temperatures a multiphase material is formed, consisting of coexisting hexagonal MnTe as well as cubic and rhombohedral $\mathrm{Ge}_{1-x} \mathrm{Mn}_{x} \mathrm{Te}$ regions. These layers display a pronounced exchange bias due to the interaction between the AFM MnTe and FM GeMnTe regions.

The $\mathrm{Ge}_{1-x} \mathrm{Mn}_{x} \mathrm{Te}$ samples were grown using a compound GeTe source and elemental $\mathrm{Mn}$ and Te beam flux sources. For all samples, the growth rate of 0.4 monolayers (ML) per second as well as the Mn concentration was precisely controlled using a quartz crystal beam flux monitor. Two series of samples were prepared, one with a Mn content of $x_{\mathrm{Mn}}$ $=0.55$ and layer thickness of $1 \mu \mathrm{m}$, and one with $x_{\mathrm{Mn}}=0.45$ and $0.5 \mu \mathrm{m}$ thickness. Within both series, the growth temperature was varied between $T_{S}=300$ to $335^{\circ} \mathrm{C}$ and an ex-

${ }^{a)}$ Electronic mail: rainer.lechner@unileoben.ac.at. cess Te flux of $0.2-0.4 \mathrm{ML} / \mathrm{s}$ was supplied during growth. The growth temperatures were carefully calibrated using an optical pyrometer with an accuracy of better than $\pm 5{ }^{\circ} \mathrm{C}$. The structural properties were determined by $\mathrm{x}$-ray diffraction (XRD), using laboratory and synchrotron sources. For complementary information, the samples were characterized by transmission electron microscopy (TEM) using a JEOL 2011 FastTEM operated at $200 \mathrm{keV}$ and the surface structure was imaged by atomic force microscopy. The magnetic properties were determined with a superconducting quantum interference device magnetometer, measuring the magnetization $M(T)$ as function of temperature $T$, as well as $M(H)$ hysteresis loops at fixed $T$ in the range of $\pm 1 \mathrm{~T}$ and $T$ $=2-350 \mathrm{~K}$. The diamagnetic background of the substrate is subtracted in these measurements.

Figure 1(a) shows the remanent magnetization $M_{\text {rem }}$ at zero field of the samples with $x_{\mathrm{Mn}}=0.55$ as a function of $T$ after field cooling (FC) from 300 to $2 \mathrm{~K}$ at $1 \mathrm{~T}$. Below $T \approx 180 \mathrm{~K}$, a nonzero magnetization appears, indicating a fer-
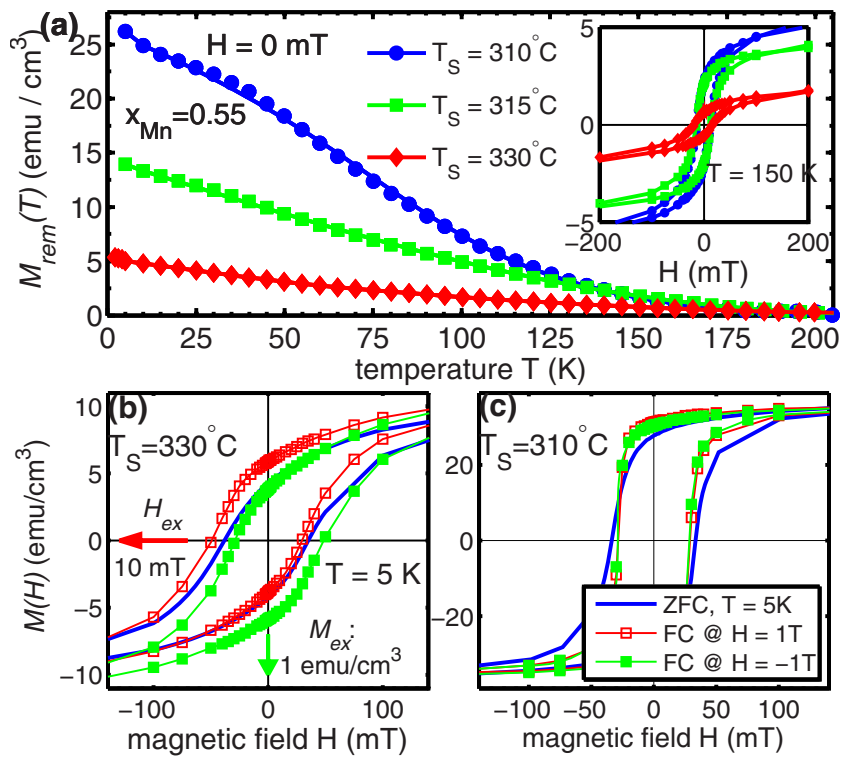

FIG. 1. (Color online) (a) Remanent magnetization $M_{\text {rem }}$ vs temperature $T$ for $\mathrm{Ge}_{1-x} \mathrm{Mn}_{x} \mathrm{Te}$ samples with $x_{\mathrm{Mn}}=0.55$ grown at different $T_{s}$ of $310^{\circ} \mathrm{C}$ (circles), $315^{\circ} \mathrm{C}$ (squares), and $335^{\circ} \mathrm{C}$ (diamonds) measured after $1 \mathrm{~T} \mathrm{FC}$. Inset: hysteresis loops at $150 \mathrm{~K}$. (b) and (c) show the hysteresis loops of the samples grown at $T_{s}=330{ }^{\circ} \mathrm{C}$, respectively, $310{ }^{\circ} \mathrm{C}$ measured at $5 \mathrm{~K}$ after ZFC (line), after +1 T FC (open squares) and $-1 \mathrm{~T} \mathrm{FC} \mathrm{(squares).}$ 
romagnetic coupling of the Mn spins. At this temperature a FM hysteresis loop with a finite coercivity is measured for all samples, as exemplified by the inset of Fig. 1(a) for T $=150 \mathrm{~K}$. Therefore, we denote this temperature as $T_{\mathrm{C}}$ although the magnetization curves do not follow the classical mean field behavior as has been already observed for $\mathrm{Ge}_{1-x} \mathrm{Mn}_{x} \mathrm{Te}$ by other groups. ${ }^{4,5} \mathrm{We}$ attribute this behavior to local fluctuations of the Mn content. However, for samples grown at higher growth temperature $T_{S}$, the remanence $M_{\text {rem }}$ at $2 \mathrm{~K}$ drastically decreases.

To check, whether additional magnetic phases exists, FC experiments were performed. In Fig. 1(b), the hysteresis loops at $5 \mathrm{~K}$ for the sample grown at $T_{s}=330{ }^{\circ} \mathrm{C}$ are shown after zero field cooling ZFC (line) and FC at +1 and $-1 \mathrm{~T}$ (red and green squares, respectively) with the magnetic field applied within the (111) plane. After positive FC, clearly a lateral shift in the hysteresis in negative field direction occurs, with an exchange field $H_{\mathrm{ex}}$ of about $10 \mathrm{mT}$, together with a vertical upward shift in the magnetization $M_{\mathrm{ex}}$ of $1 \mathrm{emu} / \mathrm{cm}^{3}$ and vice versa for negative FC. The observed lateral exchange biasing is well known for coupled FM and AFM layer systems ${ }^{11}$ and has been observed, e.g., for bilayers of FM GaMnAs coupled to AFM MnO. ${ }^{12}$ Thus, there is strong evidence for the presence of an AFM phase within this sample. The coexistence of a vertical and a lateral shift in the hysteresis loops is an indication for additional uncompensated spins pinned during FC in layered FM/AFM structures ${ }^{13}$ or in phase-separated FM systems. ${ }^{14}$ For the sample grown at low $T_{S}=310{ }^{\circ} \mathrm{C}$ depicted in Fig. 1(c), no exchange effects but only a small narrowing and a slightly increased remanence is observed in the FC measurements, which can be related to a prealignment of uncompensated spins during FC.

The presence of secondary phases in the samples grown at higher temperatures was checked by synchrotron diffraction. In the [111] XRD scans along the $q_{z}$ growth direction depicted in Fig. 2(a) (top curve) indeed, additional Bragg peaks of additional crystallographic phases are observed for the sample grown at $T_{s}=330{ }^{\circ} \mathrm{C}$. On the contrary, the sample grown at $310^{\circ} \mathrm{C}$ shows only a cubic structure as proven by the exclusive presence of the (222), (333), and (444) peaks [see lower curve of Fig. 2(a)]. In particular, no other peaks corresponding to incoherent FM precipitates like, e.g., $\mathrm{Mn}_{5} \mathrm{Ge}_{3}$ (Ref. 15) or $\mathrm{Mn}_{11} \mathrm{Ge}_{8}$ (Ref. 16) are visible. However, an asymmetric broadening of the (333) and (444) peaks at larger $q_{z}$ values is observed, which may be an indicating for a beginning formation of small amounts of additional phases. From the position of the Bragg peaks we derive a lattice constant $a_{0}$ of $5.88 \AA$ for the cubic $\mathrm{Ge}_{0.45} \mathrm{Mn}_{0.55} \mathrm{Te}$ in this sample, in good agreement with the value reported for bulk material. ${ }^{3}$ For the sample grown at a slightly higher temperature of $315{ }^{\circ} \mathrm{C}$, the cubic Bragg peaks show two shoulders on the left and right hand side, which evolve into two separate peaks for the sample grown at $330{ }^{\circ} \mathrm{C}$. The peak on the right side is identified as the (004), (006), and (008) peaks of pure hexagonal MnTe, whereas the left RLPs correspond to a rhombohedrally distorted $\mathrm{Ge}_{1-x} \mathrm{Mn}_{x} \mathrm{Te}$ phase with reduced $x_{\mathrm{Mn}}$ content. From two-dimensional RSM around the (264) RLP (not shown), the in-plane and the outof-plane lattice constants of this phase are determined, giving a lattice constant of $5.94 \AA$ and a rhombohedral distortion angle of $88.4^{\circ}$. By comparison with single phase reference
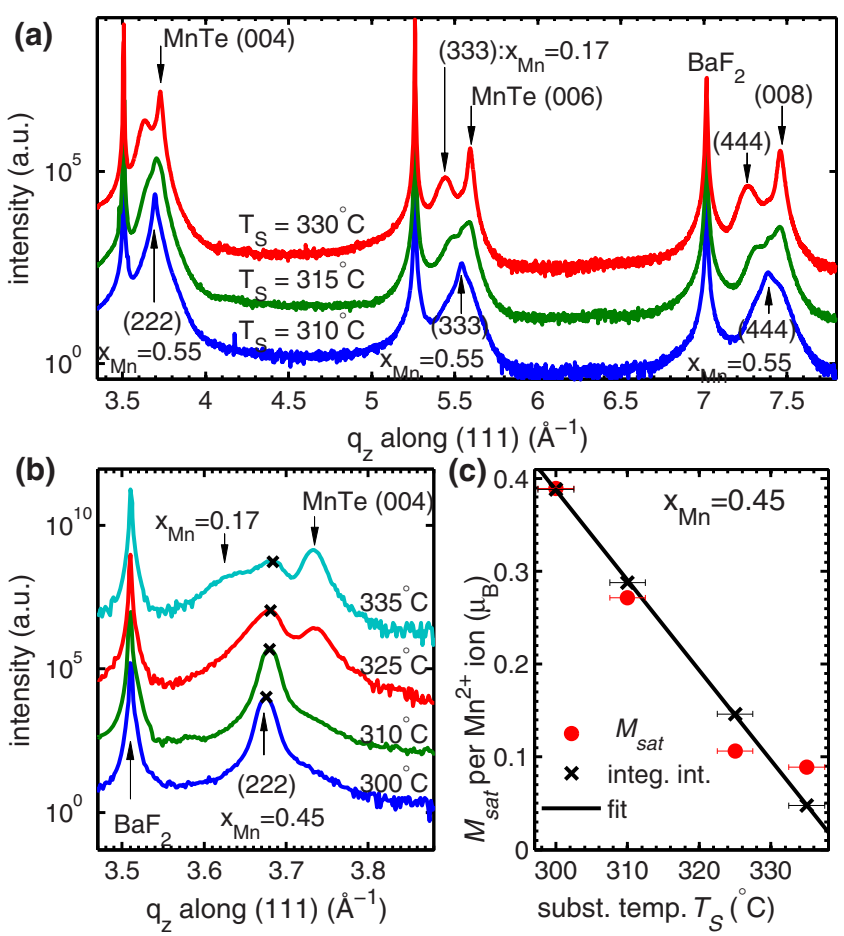

FIG. 2. (Color online) (a) XRD scan of the $\mathrm{Ge}_{1-x} \mathrm{Mn}_{x} \mathrm{Te}$ samples with $x_{\mathrm{Mn}}$ $=0.55$, grown at $T_{s}$ of $310^{\circ} \mathrm{C}, 315^{\circ} \mathrm{C}$, and $335^{\circ} \mathrm{C}$ from bottom to top, respectively. (b) XRD scan of the samples with $x_{\mathrm{Mn}}=0.45$, grown at $T_{s}$ varying from 300 to $335{ }^{\circ} \mathrm{C}$. (c) Integrated cubic (222) peak intensities $I_{\text {cub }}$ (crosses) as marked in (b) and saturation magnetization $M_{\text {sat }}$ (circles) for the samples with $x_{\mathrm{Mn}}=0.45$ plotted as a function of $T_{s}$. The black line is a linear fit to the data.

samples and bulk values, ${ }^{8}$ we derive a $x_{\mathrm{Mn}}$ value of only 0.17 for this phase.

A similar phase separation is observed for the second sample series with $x_{\mathrm{Mn}} \approx 0.45$, for which the XRD scans around the (222) RLP are shown in Fig. 2(b). Here, the onset of the formation of the hexagonal MnTe phase is found at $T_{S}=325^{\circ} \mathrm{C}$, as indicated by the appearance of the hexagonal (004) MnTe Bragg peak. The $\mathrm{Ge}_{1-x} \mathrm{Mn}_{x} \mathrm{Te}$ phase with reduced $x_{\mathrm{Mn}}=0.17$ again appears at $335^{\circ} \mathrm{C}$. The temperature for the onset of the phase decomposition between cubic $\mathrm{Ge}_{0.55} \mathrm{Mn}_{0.45} \mathrm{Te}$ and hexagonal MnTe is thus $\sim 10{ }^{\circ} \mathrm{C}$ higher as compared to the first sample series with $x_{\mathrm{Mn}}=0.55$. This is because higher $\mathrm{Mn}$ concentrations promote the formation of the MnTe phase. According to the XRD measurements, the additional phases keep the crystallographic orientation determined by the (111) $\mathrm{BaF}_{2}$ surface, i.e., the hexagonal $\mathrm{MnTe}$ $c$-axis is parallel to the growth direction and also the distorted $\mathrm{Ge}_{0.73} \mathrm{Mn}_{0.17}$ is strictly aligned along [111].

The $\mathrm{Ge}_{1-x} \mathrm{Mn}_{x} \mathrm{Te}$ sample series with $x_{\mathrm{Mn}}=0.45$ exhibits $T_{\mathrm{C}}$ values of $\sim 160 \mathrm{~K}$ and also lateral and vertical exchange coupling effects are observed for the phase separated sample. For a detailed analysis, the integrated XRD peak intensity $I_{\text {cub }}$ below the cubic (222) peak of $\mathrm{Ge}_{0.55} \mathrm{Mn}_{0.45} \mathrm{Te}$ as indicated by the crosses in Fig. 2(b) was determined and compared to the FM saturation magnetization $M_{\text {sat }}$ measured at 5 K. The results are plotted in Fig. 2(c) as a function of the growth temperature $T_{S}$, where the $I_{\text {cub }}$ values are normalized to the $M_{\text {sat }}$ value of the lowest $T_{S}$ sample. Both parameters are proportional to the total volume of the $\mathrm{FM}$ and cubic $\mathrm{Ge}_{0.55} \mathrm{Mn}_{0.45} \mathrm{Te}$ phase in the samples and indeed, both decrease linearly with the same slope with increasing $T_{S}$ [solid line in Fig. 2(c)]. The same trend of decreasing $I_{\text {cub }}$ and $M_{\text {sat }}$ 


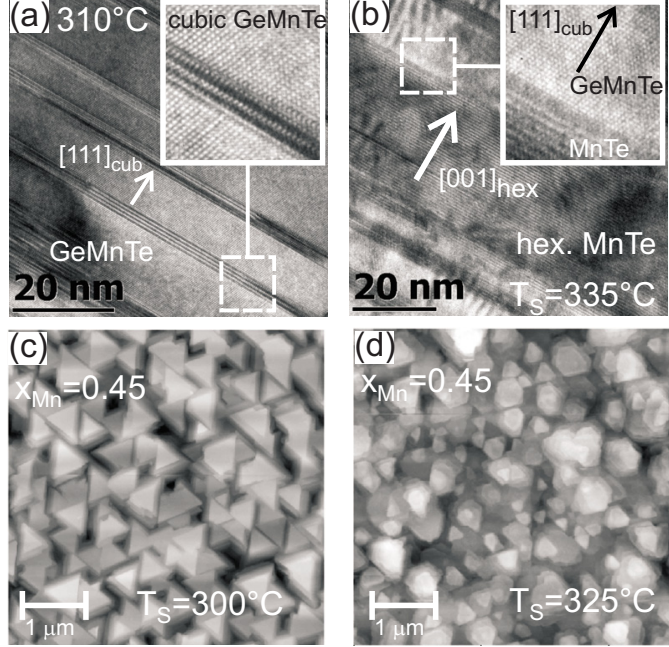

FIG. 3. (Color online) Cross-sectional TEM micrographs of $\mathrm{Ge}_{1-x} \mathrm{Mn}_{x} \mathrm{Te}$ samples with $x_{\mathrm{Mn}}=0.55$ grown at (a) $310{ }^{\circ} \mathrm{C}$ and (b) $335^{\circ} \mathrm{C}$ recorded along the [110] zone axis. Insets: Threefold magnification of the marked areas. The lower part shows atomic force microscopy images of the samples with $x_{\mathrm{Mn}}=0.45$ grown at (c) $300{ }^{\circ} \mathrm{C}$ and (d) $325^{\circ} \mathrm{C}$.

with increasing $T_{S}$ is also found for the first sample series. This is a strong indication that the observed FM behavior originates from the cubic $\mathrm{Ge}_{1-x} \mathrm{Mn}_{x} \mathrm{Te}$ phase, i.e., from $\mathrm{Mn}^{2+}$ ions incorporated on substitutional Ge lattice sites.

To get a microscopic view on the multiphase formation we investigate the single phase as well as the multiphase samples with $x_{\mathrm{Mn}}=0.55$ grown at $310^{\circ} \mathrm{C}$ and $330{ }^{\circ} \mathrm{C}$, respectively, by cross-sectional TEM. In Fig. 3(a) the cubic structure of $\mathrm{Ge}_{0.45} \mathrm{Mn}_{0.55} \mathrm{Te}$ grown at $310{ }^{\circ} \mathrm{C}$ is revealed in atomic resolution but thin cubic regions are separated along [111] by several stripes of stacking faults. Hence, the single phase sample can be better described as a stack of thin cubic $\mathrm{Ge}_{0.45} \mathrm{Mn}_{0.55} \mathrm{Te}$ slices rather than an uniform epilayer. This is also reflected by the atomic force microscopy image of the equivalent single phase $x_{\mathrm{Mn}}=0.45$ sample grown at $300{ }^{\circ} \mathrm{C}$ presented in Fig. 3(c), where two kinds of triangles are found, indicating the presence of two cubic fcc lattices rotated by $180^{\circ}$ to each other. This rotation originates from the stacking faults along the [111] growth direction. The stack of nanometer thin (111) cubic $\mathrm{Ge}_{1-x} \mathrm{Mn}_{x} \mathrm{Te}$ ferromagnets intercalated by disordered regions could also result in uncompensated spins, which can explain the observed narrowing of the hysteresis loops after FC shown in Fig. 1(c).

The $\mathrm{Ge}_{0.45} \mathrm{Mn}_{0.55} \mathrm{Te}$ sample grown at $330{ }^{\circ} \mathrm{C}$ consists of only small cubic $\mathrm{Ge}_{1-x} \mathrm{Mn}_{x} \mathrm{Te}$ regions, whereas in the majority of the sample a hexagonal structure is observed [cf TEM in Fig. 3(b)], for which the $c$-axis lattice spacing is in good agreement with the hexagonal MnTe phase. It is found that the hexagonal (001) MnTe planes are aligned parallel to the cubic (111) $\mathrm{Ge}_{1-x} \mathrm{Mn}_{x} \mathrm{Te}$ planes. The phase boundaries are not only found vertically along the growth direction but also laterally parallel to the sample surface. This is demonstrated by the atomic force microscopy image of the sample with $x_{\mathrm{Mn}}=0.45$ grown at $325{ }^{\circ} \mathrm{C}$ displayed in Fig. 3(d), where isolated triangles corresponding to cubic (111) regions are surrounded by large regions of a hexagonal surface and the lateral extent is equivalent to the separation revealed by TEM. From the TEM studies alone we cannot unambiguously distinguish the distorted $\mathrm{Ge}_{0.73} \mathrm{Mn}_{0.17}$ structure from cubic $\mathrm{Ge}_{0.55} \mathrm{Mn}_{0.45} \mathrm{Te}$. The observed phase separation be- tween $\mathrm{Ge}_{1-x} \mathrm{Mn}_{x} \mathrm{Te}$ and $\mathrm{MnTe}$ is presumably driven by a nonuniform $\mathrm{Mn}$ distribution produced at higher substrate temperatures. The formation of the rhombohedrally distorted $\mathrm{Ge}_{0.83} \mathrm{Mn}_{0.17} \mathrm{Te}$ phase could be an indication for a stable MnGeTe compound.

The coexisting hexagonal AFM MnTe and the small remains of $\mathrm{FM} \mathrm{Ge}_{1-x} \mathrm{Mn}_{x} \mathrm{Te}$ are identified as materials that magnetically couple by the exchange-bias effect as observed in Fig. 1(b). The horizontal shifts in the hysteresis loops imply a parallel coupling between the last AFM MnTe spinlayer and the adjacent $\mathrm{FM} \mathrm{Ge}_{1-x} \mathrm{Mn}_{x} \mathrm{Te}$ spins within the cubic (111) planes. The small additional vertical shifts suggest that also uncompensated MnTe spins are present at the $\mathrm{Ge}_{1-x} \mathrm{Mn}_{x} \mathrm{Te} / \mathrm{MnTe}$ interface. During FC these are aligned in field direction, parallel to the $\mathrm{FM} \mathrm{Ge} \mathrm{Ge}_{1-x} \mathrm{Mn}_{x} \mathrm{Te}$ spins. Because they are strongly coupled, i.e., pinned to the AFM $\mathrm{MnTe}$, they do not rotate with the applied field during the hysteresis measurements and thus shift the hysteresis loop in a vertical direction. Similar effects of uncompensated spins have been found also for other material systems. ${ }^{13,14}$

In conclusion, we have shown that the structural and magnetic properties of $\mathrm{Ge}_{1-x} \mathrm{Mn}_{x} \mathrm{Te}$ sensitively depends on the epitaxial growth conditions. In particular, already small changes in the growth temperature can lead to the formation of secondary phases of AFM hexagonal MnTe and distorted $\mathrm{Ge}_{1-x} \mathrm{Mn}_{x} \mathrm{Te}$ with reduced $x_{\mathrm{Mn}}$. The phase separation leads to a reduced content of the cubic FM Ge $\mathrm{Ge}_{1-x} \mathrm{Mn}_{x}$ Te phase in the layers, thus leading to a strong reduction in the total layer magnetization. Moreover, the coexistence of a FM GeMnTe and an AFM MnTe phase leads to a magnetic coupling that induces an exchange biasing of the FM regions, which is promising for possible future applications.

This work was supported by the Austrian Science Fund FWF (P18942-N20) and the FONE program (SPINTRA) of the European Science Foundation. XRD is performed at the beamline BW2 at Hasylab (Hamburg).

${ }^{1}$ G. Prinz, Science 282, 1660 (1998).

${ }^{2}$ T. Dietl and H. Ohno, Mater. Today 9, 18 (2006).

${ }^{3}$ R. Cochrane, M. Plishke, and J. Ström-Olsen, Phys. Rev. B 9, 3013 (1974).

${ }^{4}$ W. Q. Chen, S. T. Lim, C. H. Sim, J. F. Bi, K. L. Teo, T. Liew, and T. C. Chong, J. Appl. Phys. 104, 063912 (2008).

${ }^{5}$ Y. Fukuma, H. Asada, S. Miyawaki, T. Koyanagi, S. Senba, K. Goto, and H. Sato, Appl. Phys. Lett. 93, 252502 (2008).

${ }^{6}$ L. Kilanski, M. Arciszewska, W. Dobrowolski, V. Domukhovski, V. E. Slynko, and E. I. Slynko, J. Appl. Phys. 105, 103901 (2009).

${ }^{7}$ W. Knoff, V. Domukhovski, K. Dybko, P. Dziawa, M. Gorska, R. Jakiela, E. Lusakowska, A. Reszka, B. Taliashvili, T. Story, J. R. Anderson, and C. R. Rotundu, Acta Phys. Pol. A 114, 1159 (2008).

${ }^{8}$ W. Johnston and D. Sestrich, J. Inorg. Nucl. Chem. 19, 229 (1961).

${ }^{9}$ W. Szuszkiewicz, E. Dynowska, B. Witkowska, and B. Hennion, Phys. Rev. B 73, 104403 (2006).

${ }^{10}$ B. Hennion, W. Szuszkiewicz, E. Dynowska, E. Janik, and T. Wojtowicz, Phys. Rev. B 66, 224426 (2002).

${ }^{11}$ J. Nogués and I. Schuller, J. Magn. Magn. Mater. 192, 203 (1999).

${ }^{12}$ K. Eid, M. Stone, K. Ku, O. Maksimov, P. Schiffer, N. Samarth, T. Shih, and C. Palmstrøm, Appl. Phys. Lett. 85, 1556 (2004).

${ }^{13}$ P.-H. Huang, H.-H. Huang, and C.-H. Lai, Appl. Phys. Lett. 90, 062509 (2007).

${ }^{14}$ Y.-k. Tang, Y. Sun, and Z.-h. Cheng, Phys. Rev. B 73, 174419 (2006)

${ }^{15}$ R. T. Lechner, V. Holý, S. Ahlers, D. Bougeard, J. Stangl, A. Trampert, A Navarro-Quezada, and G. Bauer, Appl. Phys. Lett. 95, 023102 (2009).

${ }^{16}$ Y. Wang, J. Zou, Z. Zhao, X. Han, X. Zhou, and K. L. Wang, Appl. Phys. Lett. 92, 101913 (2008). 